\title{
Spatial Outsourcing for Location-based Services
}

\author{
Yin Yang ${ }^{\# 1}$, Stavros Papadopoulos ${ }^{\# 2}$, Dimitris Papadias ${ }^{\# 3}$, George Kollios ${ }^{* 4}$ \\ ${ }^{\#}$ Department of Computer Science and Engineering \\ Hong Kong University of Science and Technology \\ Clear Water Bay, Hong Kong \\ ${ }^{1}$ yini@cse.ust.hk \\ ${ }^{2}$ stavros@cse.ust.hk \\ dimitrisecse.ust.hk \\ *Department of Computer Science \\ Boston University \\ Boston, MA, 02215 \\ ${ }^{4}$ gkolliosdcs.bu.edu
}

\begin{abstract}
The embedding of positioning capabilities in mobile devices and the emergence of location-based applications have created novel opportunities for utilizing several types of multidimensional data through spatial outsourcing. In this setting, a data owner (DO) delegates its data management tasks to a location-based service (LBS) that processes queries originating from several clients/ subscribers. Because the LBS is not the real owner of the data, it must prove (to each client) the correctness of query output using an authenticated structure signed by the DO. Currently there is very narrow selection of multidimensional authenticated structures, among which the VR-tree is the best choice. Our first contribution is the MR-tree, a novel index suitable for spatial outsourcing. We show, analytically and experimentally, that the MR-tree outperforms the VR-tree, usually by orders of magnitude, on all performance metrics, including construction cost, index size, query and verification overhead. Motivated by the fact that successive queries by the same mobile client exhibit locality, we also propose a synchronized caching technique that utilizes the results of previous queries to reduce the size of the additional information sent to the client for verification purposes.
\end{abstract}

\section{INTRODUCTION}

The embedding of positioning capabilities (e.g., GPS) in mobile devices has triggered several types of location-based services. Such services provide fresh opportunities for data sharing and utilization. Consider a data owner (DO) that possesses a proprietary spatial dataset, such as a specialized map overlay or a set of points of interest (e.g., local businesses). The DO can profit by allowing access to the dataset. However, the cost of setting up the infrastructure, hiring qualified personnel and advertising an online service may be prohibitive. Moreover, the value of the dataset will increase if it is combined with the functionality (e.g., driving directions, aerial photos, etc.) of a general-purpose online map. These reasons provide strong motivation for outsourcing the dataset to a specialized location-based service (LBS), which achieves economy of scale by servicing multiple owners.

Outsourcing of relational databases was first proposed in [8]. In this paper, we focus on spatial outsourcing, motivated by the large availability of spatial data from various sources (e.g., satellite imagery, land surveys, environmental monitoring, traffic control). Often, agencies collecting such data (e.g., government departments, nonprofit organizations) are not able to support advanced query services; outsourcing to a LBS is the only option for utilizing the data. Furthermore, even if a DO possesses the necessary functionality, it may be beneficial in terms of cost, visibility, ease of access etc., to replicate the data in a LBS. The importance of spatial outsourcing is expected to soar with the increasing appearance of data sources and the emergence of novel mobile computing applications.

Our solutions follow the framework of Figure 1, adopted from relational database outsourcing. The DO obtains, through a key distribution center, a private and a public key. In addition to the initial data, the owner transmits to the LBS a set of signatures required for authentication. Whenever updates occur, the relevant data and signatures are also forwarded to the LBS. The LBS receives and processes spatial queries, (e.g., ranges, $k$-nearest-neighbors) from clients. Since the LBS is not the real owner of the data, the client must be able to verify the soundness and completeness of the results. Soundness means that every record in the result set is present in the owner's database and not modified. Completeness means that no valid result is missing.

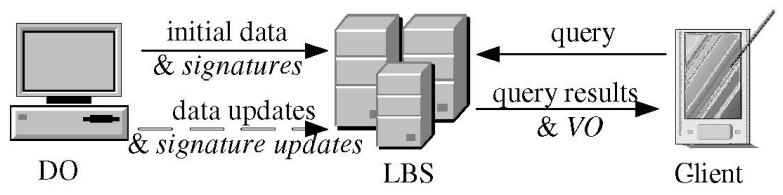

Figure 1 Database outsourcing framework

In order to process authenticated queries efficiently, the LBS indexes the data with an authenticated data structure (ADS). Each incoming query initiates the computation of a verification object (VO) using the ADS. The VO (which includes the query result) is returned to the client that can establish soundness and completeness using the public key of the DO. A crucial part in this framework concerns the ADS. Specifically, the ADS must consume little space, support efficient query processing, and lead to small VOs that can be 
easily transferred and verified. In addition, it must be able to handle updates.

Most disk-based ADSs focus on 1D ranges. The only work dealing with multi-dimensional ranges is [3], which applies the signature chain concept [16] to KD-trees and R-trees. Although the R-tree based ADS, called VR-tree, is the best between the two options, it still has some serious drawbacks: large space and query processing overhead for the LBS, high initial construction cost for the data owner, and considerable verification burden for the clients. Motivated by these problems, we propose the MR-tree, an index based on the $\mathrm{R}^{*}$ tree [2], capable of authenticating arbitrary spatial queries. We show, analytically and experimentally, that the MR-tree outperforms the VR-tree significantly on all performance metrics.

Typically, successive queries from the same client focus on a small part of the data space (e.g., a moving client asking about its surroundings). Thus, the VOs of these queries have significant overlap. Our second contribution is a synchronized caching technique that utilizes this overlap in order to reduce the size of the $V O$. Elegant algorithms continuously update the cache contents of the LBS and the client, so that they are always identical and up-to-date, without requiring any additional communication overhead. Furthermore, the space overhead for the service provider is relatively small, so that a LBS with a realistic amount of main memory (1-2 Gbytes) can support synchronized caching for millions of clients.

The rest of the paper is organized as follows. Section II surveys related work. Section III describes the basic MR-tree structure, discusses query processing, and offers cost models for its performance. Section IV focuses on the synchronized cache and its maintenance. Section $V$ contains a comprehensive experimental evaluation, and Section VI concludes the paper.

\section{RELATED WORK}

Query authentication was first studied in the Cryptography literature. The Merkle Hash Tree (MH-tree) [11] is a mainmemory binary tree that hierarchically organizes hash ${ }^{1}$ values. Figure 2 illustrates a MH-tree covering 8 data records $d_{1}-d_{8}$, each assigned to a leaf. A node $N$ contains a hash value $h_{N}$ computed as follows: if $N$ is a leaf node, $h_{N}=H\left(d_{N}\right)$, and $d_{N}$ is the assigned record of $N$, e.g., $h_{1}=H\left(d_{1}\right)$; otherwise ( $N$ is an internal node), $h_{N}=H\left(h_{N . l c} \mid h_{N . r c}\right)$, where $N . l c(N . r c)$ is the left (right) child of $N$ respectively, and "l" concatenates two binary strings, e.g., $h_{1-4}=H\left(h_{1-2} \mid h_{3-4}\right)$. After building the tree, the data owner signs the hash value $h_{\text {Root }}$, stored in the root of the MH-tree, using a public key digital signature scheme (e.g., RSA [14]).

To authenticate one-dimensional range queries, Devanbu et al. [5] sort the database records on the query attribute and index them by a MH-tree. Figure 2 shows an example, where

\footnotetext{
${ }^{1}$ Throughout the paper, the term hash function $(H)$ implies a one-way, collision-resistant hash function. In this work we employ SHA1 [14].
}

the DSP receives query $Q$ covering records $d_{4}$ and $d_{5}$. The LBS first determines the boundary records of $Q$, i.e., $d_{3}$ and $d_{6}$ which bound $Q$ 's result. Then, it follows the root-to-leaf path (Root, $N_{1-4}, N_{3-4}, N_{3}$ ) to the left boundary record $d_{3}$. For each node visited, the hash value $\left(h_{1-2}\right)$ of its left sibling is inserted into the $V O$. Records $d_{3}, d_{4}, d_{5}, d_{6}$ are added to the $V O$. Similarly, the hash values $\left(h_{7-8}\right)$ of all right-siblings on the path from the root to the right boundary $d_{6}$ are also appended. The LBS sends the $V O$ and the signature of $h_{\text {Root }}$ to the client. To verify the sequence, the client re-constructs the hash value at the root of the MHT using $d_{3}, d_{4}, d_{5}, d_{6}$ and the hash values in the $V O\left(h_{1-2}, h_{7-8}\right): h_{\text {Root }}=H\left(H\left\{h_{1-2} \mid H\left[H\left(d_{3}\right) \mid\left(H\left(d_{4}\right)\right]\right\}\right.\right.$ । $H\left\{H\left[H\left(d_{5}\right)\left|\left(H\left(d_{6}\right)\right]\right| h_{7-8}\right\}\right)$. If the reconstructed $h_{\text {Root }}$ matches the owner's signature, the result is sound. The boundary records also guarantee that no records are omitted from the query endpoints (completeness).

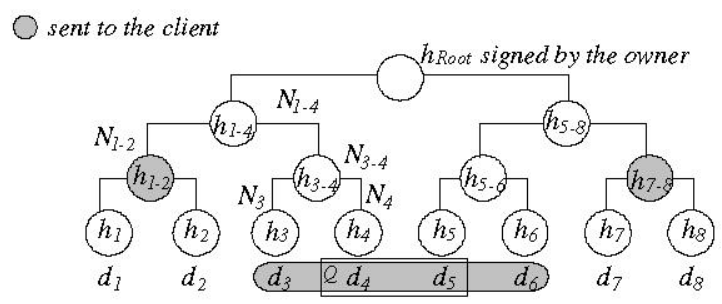

Figure 2 Example of Merkle Hash Tree

A combination of the $\mathrm{MH}$-tree and the range search tree [1] is exploited in [5] to authenticate multi-dimensional range queries. Martel et al. [12] extend the MH-tree concept to arbitrary search DAGs (Directed Acyclic Graphs), including dictionaries, tries, and optimized range search trees. Goodrich et al. [7] present ADSs for graph and geometric searching. These techniques, however, focus on main-memory and are highly theoretical in nature. For example, the range search tree is rarely used in practice due to its high space requirements: $\mathrm{O}\left(n \log ^{d-1} n\right)$, where $n$ and $d$ are the size and dimensionality of the data respectively.

The first disk-based ADS in the Database literature is the VB-tree [19], which authenticates the soundness, but not the completeness, of $1 \mathrm{D}$ range results. A subsequent signature chaining approach $[16,15]$ authenticates both soundness and completeness. Figure 3 illustrates an example, assuming that the database contains four tuples $d_{1}-d_{4}$, sorted on the search attribute. The data owner inserts two special records $d_{0}, d_{5}$ with values $-\infty$ and $+\infty$, and creates four signatures $s_{012}, s_{123}$, $s_{234}, s_{345}$, one for each triplet of adjacent tuples; $s_{012}$ corresponds to $d_{1}, s_{123}$ to $d_{2}$ and so on. The data and signatures are then transferred to the service provider.

Let the result of a range query contain $d_{1}, d_{2}$ and $d_{3}$. The service provider inserts into the $V O$ : the result $\left(d_{1}, d_{2}, d_{3}\right)$, the signature for each tuple in the result $\left(s_{012}, s_{123}, s_{234}\right)$, and the boundary records $d_{0}$ and $d_{4}$. Given the $V O$, the client checks that (i) the two boundary records fall outside the query range, and (ii) all signatures are valid. The first condition ensures that no results are missing at the range boundaries, i.e., $d_{1}$ and $d_{3}$ are indeed the first and last records of the result. The second guarantees that all results are correct. 


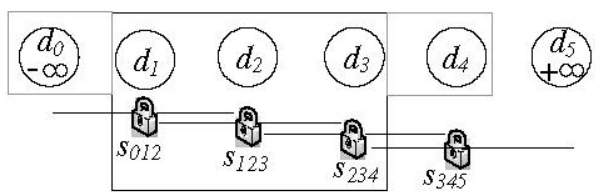

Figure 3 Example of signature chaining

The Merkle B-tree (MB-tree) [10] is a disk-based adaptation of the MH-tree. Each internal node stores entries $E$ of the form (E.p, E.k,E.h), where E.p points to a child node $N_{c}, E . k$ is the search key and $E . h$ is a hash value computed on the concatenation of the digests of the entries in $N_{c}$. Leaf nodes store records and their respective digests. The DO signs the hash value of the concatenation of the digests contained in the root of the tree. Compared to signature chaining, the MBtree incurs less space overhead since hash values are smaller than signatures and less verification effort because only the root is signed.

The only multi-dimensional ADSs in the database literature are the VKD-tree and VR-tree [3]. These structures apply the signature chain concept to KD-trees [1] and R-trees [6], respectively. We focus on the VR-tree since, as shown in [3], it outperforms the VKD-tree. All points in a leaf node are sorted according to their $x$-coordinates. Two fictitious points are added before the first and after the last point of the node. Following [16], the VR-tree creates one signature for each sequence of three points and stores it along with each entry, e.g., in Figure $4 \mathrm{a}$, the entry for $P_{8}$ contains $s_{789}$. For internal nodes, the minimum bounding rectangles (MBRs) of child nodes are sorted on their left side and a signature chain is formed in a similar way. For instance, in Figure $4 b$, the signature of node $N_{4}$ is $s_{345}$.

The processing of range queries is similar to the R-tree, except for the additional $V O$ construction. Consider the window query $Q$ in Figure $4 \mathrm{a}$, which retrieves $P_{9}$ and $P_{11}$. For each index node visited, all MBRs in this node are inserted into the $V O$. The corresponding signatures participate in the incremental construction of an aggregated $d^{2}$ signature $s$. When a leaf node of the VR-tree is reached, all points whose $x$ coordinates fall in the query range $\left(P_{8}-P_{12}\right)$ and the two boundary points $\left(P_{7}, P_{13}\right)$ are inserted into the $V O$. The corresponding signatures are aggregated in $s$, which is included in the $V O$.

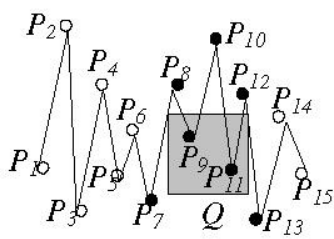

(a) Leaf Node (b) Internal Node

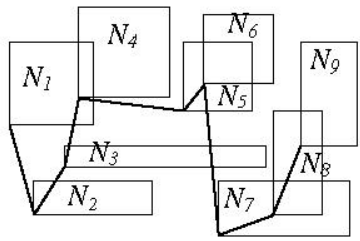

Figure 4 Signature chains in the VR-tree

\footnotetext{
${ }^{2}$ Signature aggregation [13] condenses multiple signatures
} into a single one, thus significantly reducing the total size.
To verify results, the client starts from the root and compares all MBRs against the query. Then, it reads the content of each node whose MBR overlaps the query from the $V O$ and recursively checks all its children. Finally, at the leaf level, it can extract the query results. During this procedure, the client incrementally constructs an aggregated digest from the MBRs and points included in the $V O$, which is eventually verified against the aggregated signature. As we show, analytically and experimentally, the VR-tree has some serious shortcomings: large space and query processing overhead, high initial construction cost, and considerable verification burden for the clients. The MR-tree, discussed next, aims at solving these problems.

\section{MR-TREE}

Section III-A presents the structure of the MR-tree, and describes query processing and authentication. Section III-B contains cost models for various performance metrics, and compares the MR-tree and the VR-tree analytically.

\section{A. Structure and Query Processing}

The MR-tree combines concepts from MB- [10] and R*trees [2]. Figure 5 illustrates the node structure. Leaf nodes are identical to those of the $\mathrm{R}^{*}$-tree: each entry $R_{i}$ corresponds to a data object. Note that although our examples use points, the MR-tree is applicable to objects with arbitrary shapes. A hash value is computed on the concatenation of the binary representation of all objects in the node. Internal nodes contain entries of the form $\left(p_{i}, M B R_{i}, h_{i}\right)$, signifying the pointer, minimum bounding rectangle and hash value of the $i$ th child, respectively. The hash value summarizes child nodes' MBRs $\left(M B R_{l}-M B R_{f}\right)$, in addition to their hash values $\left(h_{l}-h_{f}\right)$. The hash value of the root node $h_{\text {root }}$ is signed by the data owner and stored with the tree. The MR-tree supports updates based on the corresponding algorithms of the $\mathrm{R}^{*}$-tree. When a node changes (due to an insertion or deletion), the corresponding hash value in the parent entry is updated recursively, until reaching the root. The owner then signs the new root and transmits the changes to the LBS.

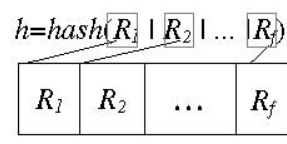

(a) Leaf Node

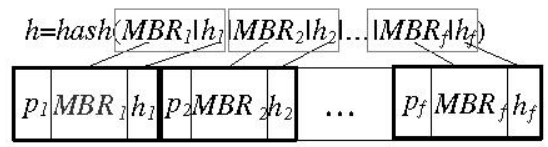

(b) Internal Node
Figure 5 MR-tree node structure

To process a range query $Q$, the LBS invokes Range Query(root, $Q$ ), shown in Figure 6. The algorithm computes the verification object by following a depth-first traversal of the MR-tree. The $V O$ contains three types of data: (i) all objects in each leaf node visited (Line 4), (ii) the MBR and hash values of pruned nodes (Line 7), and (iii) special tokens [ and ] that mark the scope of a node (Lines 1 and 8). New entries are always appended to the end of the $V O$.

Consider, for instance, query $Q$ in the example tree of Figure 7. Similar to conventional R-trees, RangeQuery starts from the root and visits recursively all entries that overlap the 
shaded rectangle: $N_{1}, N_{4}, N_{2}, N_{5}$. After termination, the verification object is: [[(MBR_N$N_{3}$, hash $\left.\left.\left.\_N_{3}\right),\left[P_{4}, P_{5}, P_{6}\right]\right]\right]$, $\left[\left[P_{7}, P_{8}, P_{9}\right]\right.$, (MBR $\_N_{6}$, hash $\left.\left.\left.\_N_{6}\right)\right]\right]$. The tokens signify the contents of a node; for instance, the component [[(MBR $N_{3}$, hash $\left.\left.\left.\_N_{3}\right),\left[P_{4}, P_{5}, P_{6}\right]\right]\right]$ corresponds to the first root entry $\left(N_{1}\right)$, and the rest of the $V O$ to the second one $\left(N_{2}\right)$. The LBS transmits the $V O$ and the root signature $s_{\text {root }}$ to the client. Note that the actual result (e.g., $P_{4}, P_{7}$ ) is part of the $V O$.

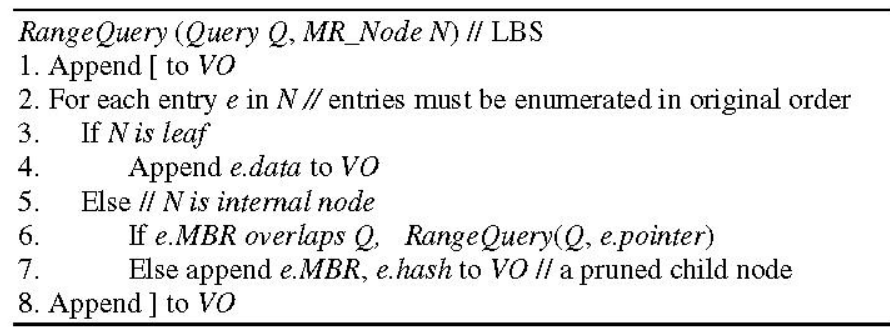

Figure 6 Range query processing with the MR-tree

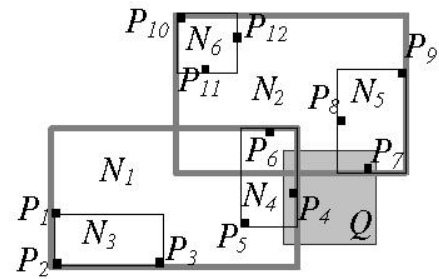

(a) Points and Node MBRs

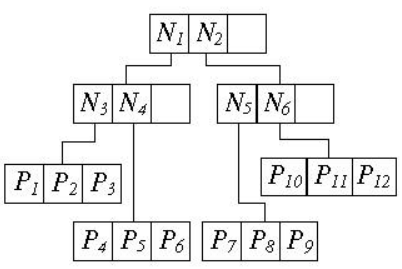

(b) MR-tree
Figure 7 Example range query

To verify the query results, the client first scans the $V O$ to check that: (i) each data point in the $V O$ is either outside $Q$, or included in the result set, (ii) no MBR (of a pruned node) in the $V O$ overlaps $Q$, and (iii) the computed $h_{\text {root }}$ from the $V O$ agrees with $s_{\text {root }}$. Figure 8 shows the recursive procedure RootHash that computes $h_{\text {root }}$. The main idea is to simulate the MR-tree traversal performed by the LBS, and calculate the MBR and hash values bottom-up.

(MBRValue, HashValue) RootHash(VO) // Client

1. Initialize str, $M B R$ to empty string and MBR value respectively

2. While $V O$ still has entries

3. Get next entry $e_{V}$ from $V O$

4. If $e_{V}$ is ], go to Line $13 / /$ break the while-loop

5. If $e_{V}$ is a data object $R$

6. If $R$ overlaps the query, Add $R$ to the result set

7. $\quad M B R \_c=$ the MBR of $R$

8. $s t r \_c=$ the binary representation of $R$

9. If $e_{V}$ is [, $\left(M B R \_c\right.$, hash_c) $=$ RootHash $(V O)$

10. If $e_{Y}$ is a pair of MBR/hash value (MBR_e $e_{Y}$, hash $\left.e_{Y}\right)$

11. $M B R \_c, s t r \_c=\left(M B R \_e_{y}, h a s h \_e_{y}\right)$

12. Enlarge $M B R$ to include $M B R \_c$

13. Concatenate $s t r$ with $s t r_{-} c$

14. Return (MBR, hash (str))

Figure 8 Algorithm for re-computing $h_{\text {root }}$

In the example of Figure 7, RootHash computes the MBR and hash value of nodes $N_{4}$ (from $P_{4}-P_{6}$ ), $N_{1}$ (from $N_{3}, N_{4}$ ), $N_{5}$ (from $P_{7}-P_{9}$ ), $N_{2}$ (from $N_{5}, N_{6}$ ), root (from $N_{1}, N_{2}$ ), in this order. Note that all entries in the $V O$, from the [ of the root to its ], must be used. Furthermore, the algorithm is online, meaning that it performs a single sequential scan of the $V O$. During the verification, the actual results $\left(P_{4}, P_{7}\right)$ are extracted in Line 6. In addition, the client receives some objects $\left(P_{5}, P_{6}\right.$, $\left.P_{8}, P_{9}\right)$ in the $V O$, which are not part of the result. Pang et. al. [16] propose a solution for avoiding disclosure of such objects, when the outsourced database must comply with certain access control policies. In this work, we consider that clients can issue queries freely without constraints. Nevertheless, the solution of [16] can be applied in conjunction with the proposed methods to hide the additional objects, if necessary.

Proof of soundness: Assume that an object $P$ in the result set is bogus or modified. Because the hash function is collisionresistant and $P$ must be used by RootHash, the re-computed $h_{\text {root }}$ can not be verified against $s_{\text {root }}$, which is detected by the client.

Proof of completeness. Let $P$ be an object satisfying $Q$. Consider the leaf node $N_{l}$ containing $P$. For the re-computed $h_{\text {root }}$ to match $s_{\text {root }}$, either $N_{l}$ 's true contents or MBR/hash must be in the $V O$. In the former case $P$ is in the $V O$, and extracted in Line 6 of RootHash. In the latter case, $N_{l}$ 's MBR overlaps $Q$, which alarms the client about potential violation of completeness.

In addition to range search, the MR-tree can authenticate other common spatial queries, including $k$ nearest neighbors $(k \mathrm{NN})$ and skylines. Given a point $Q$, a $k \mathrm{NN}$ query retrieves the $k$ points from the data set that are closest to $Q$ [9]. In the example of Figure $9 \mathrm{a}$, the three NNs of $Q$ are $P_{1}, P_{2}$ and $P_{3}$, in increasing order of distance from $Q$. A key observation is that the $k \mathrm{NN}$ of $Q$ lie in a circular area $C$ centered at $Q$ that contains exactly $k$ data points. Therefore, the LBS can prove the $k \mathrm{NN}$ results by sending to the client the $V O$ corresponding to $C$. Specifically, it first finds the $k$ neighbors, then it computes $C$, and finally executes RangeQuery treating $C$ as the range. The verification process of the client is identical to the one performed for range queries.

A skyline query retrieves all points that are not dominated by others in the dataset [18]. A point $P_{i}$ dominates another $P_{j}$, if and only if, the co-ordinate of $P_{i}$ on each dimension is no larger than the corresponding co-ordinate of $P_{j}$. The skyline in Figure $9 \mathrm{~b}$ contains $P_{1}, P_{2}$ and $P_{7}$. To prove it, the LBS processes a range query that contains the area of the data space not dominated by any skyline point. This area (shaded in Figure 9b) can be divided into multiple rectangles. The result contains only the skyline points, and can again be verified according to the methodology of range search.

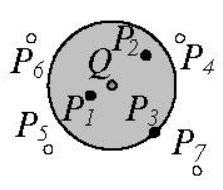

(a) $k \mathrm{NN}$

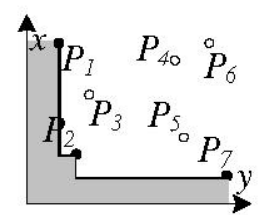

(b) Skyline
Figure 9 Alternative queries 


\section{B. Cost Models}

The important performance metrics for authenticated structures are (i) index construction time, (ii) index size, (iii) query processing cost, (iv) size of the $V O$, and (v) verification time. The first metric affects the party that builds the index, i.e., depending on the system, the DO or the LBS. The second one burdens the LBS and, in some cases, the DO (if it also has to maintain the index). Furthermore, it affects the communication cost between the two. Metric (iii) is important only for the LBS. The size of the $V O$ influences the network overhead between the LBS and the client. Finally, the verification time burdens exclusively the client. In the sequel we compare analytically the MR-tree and the VR-tree on the above metrics. Table I summarizes the symbols used in the analysis, as well as their typical values $\left(1 \mathrm{msec}=10^{-3}\right.$ seconds, $1 \mu \mathrm{sec}=10^{-6}$ seconds). These values were obtained based on the hardware and software settings of our experiments, using the Crypto++ library. Our measurements are similar to those of the library benchmarks [4] and the values suggested in [10].

TABLE I

SYMBOLS AND VALUES IN THE ANALYSIS

\begin{tabular}{|c|c|c|}
\hline Symbol & Meaning & Typical Value \\
\hline$C_{s}$ & CPU cost of sign operation & $3.4 \mathrm{msec}$ \\
\hline$C_{\nu}$ & CPU cost of verify operation & $160 \mu \mathrm{sec}$ \\
\hline$C_{h}$ & CPU cost of hash operation & $28 \mu \mathrm{sec}$ \\
\hline$\overline{C_{m}}$ & CPU cost of multiply operation & $43 \mu \mathrm{sec}$ \\
\hline$C_{N A}$ & CPU cost of a random node access & $15 \mathrm{msec}$ \\
\hline$S_{s}$ & size of a signature & 128 bytes \\
\hline$S_{h}$ & size of a hash value & 20 bytes \\
\hline$S_{M}$ & size of an MBR & 32 bytes \\
\hline$S_{y}$ & size of a data point & 16 bytes \\
\hline$n$ & data cardinality & $2,000,000$ \\
\hline$d$ & data dimensionality & 2 \\
\hline$Q_{l}$ & query extent on one dimension & $10 \%$ of space \\
\hline$b$ & block size & 4096 bytes \\
\hline$f_{l}$ & fanout of leaf node & \begin{tabular}{l|l} 
VR 19 & MR 179 \\
\end{tabular} \\
\hline$f_{n}$ & fanout of internal node & \begin{tabular}{l|l} 
VR 17 & MR 51 \\
\end{tabular} \\
\hline$h$ & height of the tree & MR 4 \\
\hline
\end{tabular}

We first establish a simple cost model for the R-tree, based on the fact that in $d$-dimensional unit space $[0,1]^{d}$, the probability that two random rectangles $r_{1}, r_{2}$ overlap is:

$$
P_{\text {overlap }}\left(r_{1}, r_{2}\right)=\prod_{j=1}^{d}\left(r_{1} l_{j}+r_{2} . l_{j}\right)
$$

where $r . l_{j}$ denotes rectangle $r$ 's extent along the $j$ th dimension [17]. For simplicity, we assume that the data set contains points (rectangular data are discussed in [21]) uniformly distributed in the unit space and query $Q$ has equal length $Q_{l}$ on all dimensions. Let $f_{l}\left(f_{n}\right)$ be the average fanout of a leaf (internal) node, and $n$ be the data cardinality. The number of leaf nodes is $n / f$, and the height of the R-tree is $h=1+\left\lceil\log _{f_{n}}\left(n / f_{l}\right)\right\rceil$. The number of internal nodes at depth $i$ of the tree (assuming a complete tree where the root has depth 0 ) is $f_{n}^{i}$, each containing $n / f_{n}^{i}$ data points in its subtree. Because of the uniform distribution, the number of points in a node is proportional to the space covered by this node. Following [21], we assume that all nodes at the same level are squares with similar sizes. Therefore, a node at depth $i$ covers $1 / f_{n}^{i}$ space, and has length $\sqrt[d]{1 / f_{n}^{i}}$ on each dimension. Applying Equation 3.1, the total cost of processing $Q$ using the VR- or the MR-tree is:

$$
C_{Q}=C_{N A}\left(\sum_{i=0}^{h-2} f_{n}^{i}\left(\sqrt[d]{1 / f_{n}^{i}}+Q_{l}\right)^{d}+f_{l}\left(\sqrt[d]{f_{l} / n}+Q_{l}\right)^{d}\right)
$$

where $C_{N A}$ is the cost of a node access. Similarly, the storage overhead of both the VR- and the MR-tree can be estimated by:

$$
S_{\text {index }}=b\left(\sum_{i=1}^{h-2} f_{n}^{i}+n / f_{l}\right)
$$

where $b$ is the block size. The difference between the two structures regards the authentication information, leading to different fanouts $\left(f_{l}, f_{n}\right)$. The VR-tree maintains one signature (128 bytes) per entry in every node (leaf or internal). In contrast, the MR-tree adds hash values ( 20 bytes each) only to internal nodes. Assuming a page of 4 KBytes, $70 \%$ average storage utilization and double precision, the VR-tree has a fanout of $f_{l}=19$ (leaf) and $f_{n}=17$ (internal), while for the MRtree $f_{l}=179$ and $f_{n}=51$. The lower fanout of the VR-tree increases its height.

Besides R-tree generation, the VR-tree requires a signature for each object and node. The MR-tree only involves cheap computations of hash values for nodes (but not objects). If the cost of a sign / verify / hash operation is $C_{s}, C_{v}, C_{k}$ respectively, the initial construction overhead of the VR-tree (MR-tree) is given by equation 3.4 (3.5):

$$
\begin{aligned}
& C_{\text {init }}^{\text {VR }}=C_{s}\left(\sum_{i=1}^{h-1} f_{n}^{i}+n\right) \\
& C_{\text {inat }}^{M R}=C_{s}+C_{h} \sum_{i=0}^{h-1} f_{n}^{i}
\end{aligned}
$$

Let the size of a signature, an MBR, a hash value and a data point be $S_{s}, S_{M}, S_{h}$ and $S_{p}$, respectively. Then, the $V O$ of the VR-tree with signature aggregation consumes space:

$$
S_{V O}^{V R}=S_{s}+\sum_{i=0}^{h-2} f_{n}^{i+1}\left(\sqrt[d]{1 / f_{n}^{i}}+Q_{l}\right)^{d} S_{M}+n\left(\sqrt[d]{f_{l} / n}+Q_{l}\right)^{d} S_{p}
$$

where the last two terms estimate MBRs and points for visited internal and leaf nodes respectively. Note that with signature aggregation, there is a single signature, thus the $V O$ size is relatively small. To prepare this $V O$, however, the LBS must perform modular multiplications, whose cost is:

$$
C_{V O}^{V R}=C_{m}\left(\sum_{i=0}^{h-2} f_{n}^{i+1}\left(\sqrt[d]{1 / f_{n}^{i}}+Q_{l}\right)^{d}+n\left(\sqrt[d]{f_{l} / n}+Q_{l}\right)^{d}\right)
$$

Thus, the total query processing overhead for the VR-tree is the sum of the two costs expressed in Equations 3.2 and 3.7. The $V O$ size of the MR-tree is given by Equation 3.8. The complicated part is to analyze the total number of pruned nodes during query processing. $P N(i)$ estimates the number of pruned nodes at depth $i$, by computing the number of nodes outside $Q$, subtracted by descendents of higher pruned nodes.

$$
\begin{aligned}
& S_{V O}^{M R}=\sum_{i=0}^{h-1} P N(i)\left(S_{h}+S_{M}\right)+n\left(\sqrt[4]{f_{l} / n}+Q_{l}\right)^{d} S_{p} \\
& P N(i)=f_{n}^{i}\left(1-\left(\sqrt{1 / f_{n}^{i}}+Q_{l}\right)^{d}\right)-\sum_{i=0}^{i-1} P N(j) f_{n}^{i-j}
\end{aligned}
$$


Finally we estimate the verification time for the client, which is dominated by modular multiplications (VR-tree) or computing hash values (MR-tree). The costs of the VR-tree (with signature aggregation) and the MR-tree are given by Equations 3.9-3.10. The MR-tree has a clear advantage because (i) for each node, the MR-tree invokes the hash function once, whereas the VR-tree performs modular multiplication for each entry, and (ii) $C_{\hbar}<C_{m}$.

$$
\begin{gathered}
C_{\text {Client }}^{V R}=C_{v}+C_{m}\left(\sum_{i=0}^{h-2} f_{n}^{i+1}\left(\sqrt{1 / f_{n}^{i}}+Q_{i}\right)^{d}+n\left(\sqrt[d]{f_{l} / n}+Q_{l}\right)^{d}\right) \\
C_{\text {Client }}^{M R}=\sum_{i=0}^{h-1} f_{n}^{i}\left(\sqrt[d]{1 / f_{n}^{i}}+Q_{l}\right)^{d} C_{h}+C_{v}
\end{gathered}
$$

Table II shows the costs calculated by the above equations using the typical values of Table I. The VR-tree incurs about 30 times the overhead of the MR-tree for computing the authentication information (in the entire tree), and is 8 times larger. The MR-tree is also significantly better in terms of query processing and verification cost. The latter is particularly important because the clients are mobile devices with limited computing power. The only aspect where the two structures are similar is $V O$ size. Next, we present an optimization for reducing the $V O$.

TABLE II

COMPARISON OF ESTIMATED COSTS

\begin{tabular}{|c|c|c|}
\hline Costs & MR-tree & VR-tree \\
\hline Time for Computing Authentication Data & $4 \mathrm{sec}$ & 2 hours \\
\hline Index Size & $57 \mathrm{MBytes}$ & 511 MBytes \\
\hline Query Processing Time & $2 \mathrm{sec}$ & $22 \mathrm{sec}$ \\
\hline$V O$ size (bytes) & $390 \mathrm{KBytes}$ & $398 \mathrm{KBytes}$ \\
\hline Verification (CPU time) & $41 \mathrm{~ms}$ & $991 \mathrm{~ms}$ \\
\hline
\end{tabular}

\section{SYNCHRONIZED CACHING FOR THE MR-TREE}

Each client is expected to issue numerous queries at different times. The $V O$ of these queries always share common entries, specifically, $s_{\text {root }}$ and the MBR/hash values of the root nodes (since the root is always accessed). In practice, the overlap is significantly larger because most queries focus on a small part of the data space. For instance, a moving client is likely to ask about its surroundings at successive locations that are close to each other. Assume that the client maintains the $V O$ of previous queries in a cache. When the LBS processes a new query, it needs to send only the part of the $V O$ that is not already in the cache. However, in order for this optimization to become possible, the LBS must have complete knowledge of the client's cache. We propose a synchronized cache $(S C)$ scheme, where the LBS maintains, for each client, an abstract copy of its cache. The term abstract means that concrete hash values and records are replaced with placeholders (to be discussed shortly). As shown in the experimental evaluation, a LBS with a reasonable amount of main memory can support synchronized caching for millions of clients.

Figure 10 summarizes the proposed framework for synchronized caching. Given a query from the client, the LBS computes the (uncompressed) $V O_{\text {raw. }}$. Then, it applies an algorithm (ReduceVO) that utilizes the contents of the $S C$ to derive a compressed $V O_{\text {reduced, }}$, i.e., the part of $V O_{\text {raw }}$ that is not in $S C$. $V O_{\text {reduced }}$, which is usually much smaller than $V O_{\text {raw }}$, is sent to the client. The client restores $V O_{\text {raw }}$ (using the reverse process of ReduceVO) and uses it to verify the query result. Both the LBS and the client incorporate the content of $V O_{\text {reduced }}$ to the $S C$ through $M$ ergeVO algorithm. The addition of new content may increase the size of the $S C$ beyond a predefined limit. In this case, PurgeSC frees space by expunging "old" data. It is easy to verify that if the LBS and the client start with an empty $S C$ and have the same space limit, then their cache contents are identical at all times. Thus, there is no additional communication overhead for cache synchronization. On the other hand, this optimization minimizes the $V O$ size and the associated transmission cost.

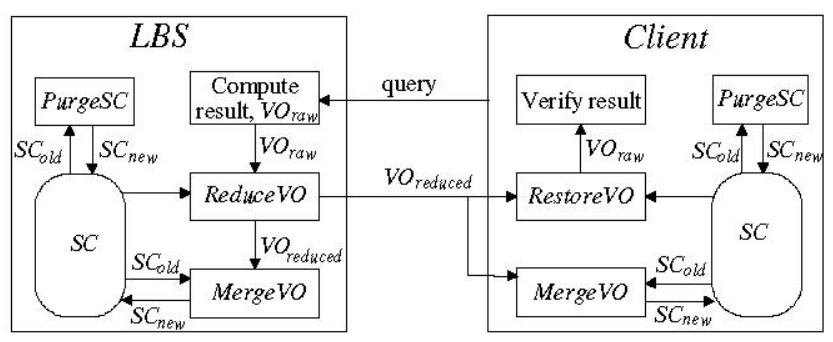

Figure 10 Framework of synchronized caching

Section IV-A describes the $V O$ minimization process (i.e., ReduceVO), while Section IV-B focuses on the $S C$ maintenance (i.e., MergeVO and PurgeSC). In our discussion, we distinguish between value and token entries in the VO. A value entry is a data point, a pair of MBR/hash values, or the signature $s_{\text {root }}$. A token is [ or ].

\section{A. Minimizing the Size of the VO}

Similar to the $V O$, the $S C$ is a linked list of value and token entries except that the copy maintained by the LBS uses a placeholder (i) for each (MBR, hash value) pair and (ii) for all records in a leaf node. Moreover, each token [ is associated with a timestamp to be discussed later. We use the running example of Figure 11, where a client asks two queries $Q_{1}, Q_{2}$. The $S C$ is initialized to be empty. When the first query is processed, its $V O$ is copied to the caches of both the LBS and the client. After this step, the $S C$ equals $V O\left(Q_{1}\right)=\left[\left[\left[P_{1}, P_{2}\right.\right.\right.$, $\left.P_{3}\right],\left(\operatorname{MBR} \_N_{4}\right.$, hash $\left.\left.\_N_{4}\right)\right]$, (MBR $\_N_{2}$, hash $\left.\left.\_N_{2}\right)\right], s_{\text {root }}$.

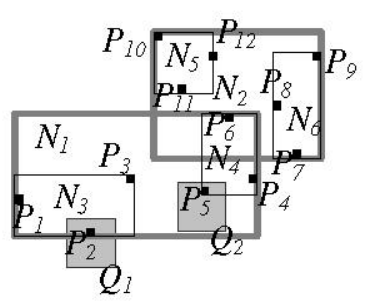

\begin{tabular}{|c|c|}
\hline Query & VO \\
\hline$Q_{I}$ & $\begin{array}{c}{\left[\left[\left[P_{1}, P_{2}, P_{3}\right],\right.\right.} \\
\left.\left(\text { MBR_N } N_{4} \text {, hash_ } N_{4}\right)\right], \\
\left.\left.\text { (MBR__ } N_{2} \text {, hash_ } N_{2}\right)\right], \\
\boldsymbol{s}_{\text {root }}\end{array}$ \\
\hline$Q_{2}$ & $\begin{array}{c}{\left[\left[\left(\mathrm{MBR} \_N_{3}, \text { hash_N} N_{3}\right),\right.\right.} \\
\left.\left[P_{S}, P_{6}\right]\right],\left(\mathbf{M B R} N_{2},\right. \\
\left.\left.\text { hash_N } N_{2}\right)\right], s_{\text {root }}\end{array}$ \\
\hline
\end{tabular}

(a) Queries
Reduced $V O\left(Q_{2}\right): \quad\left[\left[S C_{-}\right.\right.$compute, $\left.\left[P_{5}, P_{6}\right]\right], S C_{-}$hit $], S C_{-}$hit

(b) $V O \mathrm{~s}$
Figure 11 Queries with overlapping VOs 
When later the LBS processes $Q_{2}$, it compares $V O\left(Q_{2}\right)=$ [[(MBR $\_N_{3}$, hash $\left.\left.\_N_{3}\right),\left[P_{5}, P_{6}\right]\right], \quad\left(\operatorname{MBR} \_N_{2}\right.$, hash $\left.\left.\_N_{2}\right)\right], s_{\text {root }}$ with the $S C$. (MBR $\_N_{2}$, hash $\_N_{2}$ ) and $s_{\text {root }}$ are in the $S C$ and replaced with a token $S C$ hit, reducing the $V O$ size from 5 to 3 value entries. Moreover, the entire sub-tree of $N_{3}\left(P_{1}-P_{3}\right)$ is in the $S C$, meaning that the client is able to compute MBR $N_{3}$ and hash $N_{3}$. Therefore, the LBS substitutes the entry (MBR $N_{3}$, hash $\_N_{3}$ ) with a token $S C \_$compute, leading to a $V O\left(Q_{2}\right)$ with only 2 value entries $P_{5}$ and $P_{6}$.

Figure 12 shows ReduceVO, which utilizes the $S C$ to minimize the verification object. Let $V O_{\text {raw }}\left(V O_{\text {reduced }}\right)$ be the $V O$ before (after) the shrinking process. ReduceVO scans the $S C$ and $V O_{\text {raw }}$ in parallel, computing $V O_{\text {reduced. }}$ Each step retrieves an entry $e_{V}\left(e_{S}\right)$ from $V O_{\text {raw }}(S C)$. An important invariant is that $e_{V}$ and $e_{S}$ must always correspond to the same node (or data record) in the MR-tree. We illustrate the algorithm using the example of Figure 11 and assuming $S C=$ $V O\left(Q_{1}\right)$ and $V O_{\text {raw }}=V O\left(Q_{2}\right)$. In the first two steps, $e_{S}$ and $e_{V}$ are both [ (Case 4), and the LBS simply appends two [ into $V O_{\text {reduced. }}$. Then, $e_{S}$ becomes [ and $e_{V}=\left(\operatorname{MBR} \_N_{3}\right.$, hash $\left.\_N_{3}\right)$ (Case 2). Both $e_{S}$ and $e_{V}$ refer to the same node $N_{3}$ : the $S C$ contains details about $N_{3}$, whereas the $V O$ only contains aggregates (i.e., MBR and hash). Therefore, it is possible to compute $e_{V}$ with $S C$ entries starting from $e_{S}$ until its corresponding ], i.e., $\left[P_{1}, P_{2}, P_{3}\right]$. Thus the LBS appends an $S C \_$compute token to $V O_{\text {reduced }}$. Note that we must adjust the current entry of $S C$ accordingly (Line 8-10) to ensure the invariant stated above. Next, $e_{V}$ becomes [ (before $P_{5}$ ) and $e_{S}$ is (MBR $\_N_{4}$, hash $\_N_{4}$ ) (Case 3). Conversely to Case 2, now the $V O_{\text {raw }}$ contains details (i.e., $\left[P_{5}, P_{6}\right]$ ) while the $S C$ contains aggregates. Starting from this [, the LBS copies everything from $V O_{\text {raw }}$ to $V O_{\text {reduced }}$, until the corresponding ] is reached. Then, both $e_{V}$ and $e_{S}$ become successively [ (Case 4), and (MBR $\_N_{2}$, hash $\_N_{2}$ ) (Case 1). The token $S C$ h hit is appended to $V O_{\text {reduced }}$. Finally, for $s_{\text {root }}, S C$ hit is appended to $V O_{\text {reduced }}$. $V O_{\text {reduced }}$ is sent to the client, which restores the original $V O_{\text {raw }}$ (following the reverse process of ReduceVO) and uses it to verify the query results.

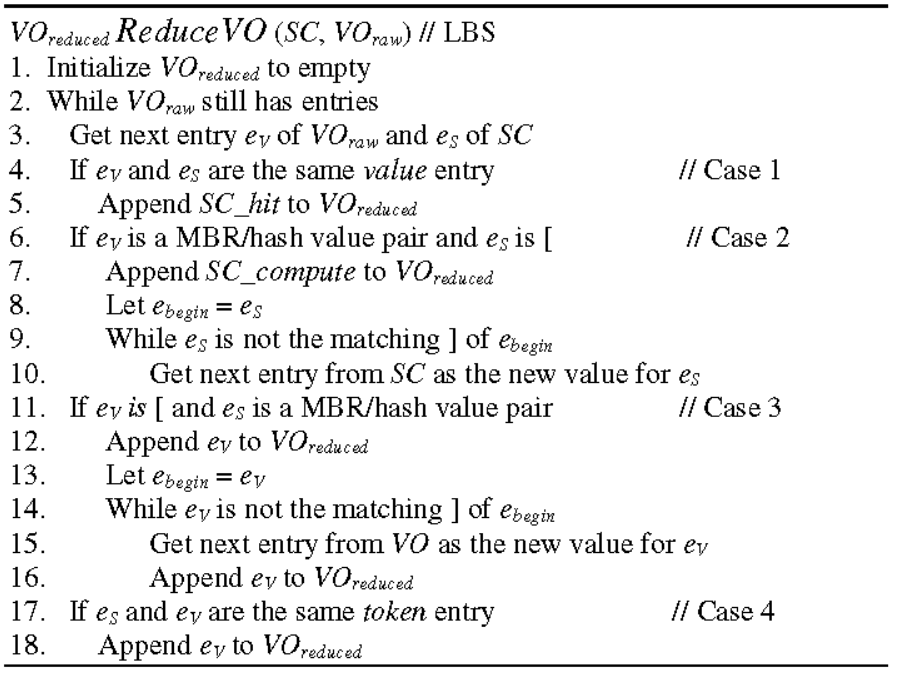

Figure 12 ReduceVO algorithm

\section{B. Updating the $\mathrm{SC}$}

Every new $V O_{\text {reduced }}$ updates the $S C$ at the LBS and the client. Specifically, both LBS/client integrate $V O_{\text {reduced }}$ into the $S C$ using MergeVO, shown in Figure 13. The $S C$ before (after) this operation is called $S C_{\text {old }}\left(S C_{\text {new }}\right)$. Initially, $S C_{\text {new }}$ is empty. Each step of MergeVO retrieves pairs of entries $e_{V} \in$ $V O_{\text {reduced }}$ and $e_{S} \in S C_{\text {old }}$ in parallel. Depending on the type of these entries, we have 4 cases, similar to ReduceVO. Case 1 occurs when $e_{V}$ is a hit for $e_{S} ; e_{S}$ is added to $S C_{\text {new }}$ and its [ receives a timestamp equal to the current time. As we discuss shortly, timestamps are used to expunge old entries according to an LRU policy. Case 2 happens when $e_{V}$ can be computed by $e_{s}$. MergeVO inserts to $S C_{\text {new }}$ all entries between the [ and ] tokens of $e_{s}$. The recency of these entries is not updated, because $S C \_c o m p u t e$ implies that only the aggregates, but not the actual contents, of $e_{S}$ are required for the query. Case 3 incorporates new information from the $V O$ into $S C_{\text {new. }}$. Specifically, when the $V O$ contains details of an MR-tree node while $S C_{\text {old }}$ has only aggregates, we append these details into $S C_{\text {new. }}$. In the example of Figure 11, if the client has $S C_{\text {old }}=$ $V O\left(Q_{1}\right)$ and receives the reduced $V O\left(Q_{2}\right)$, MergeVO updates the timestamps and replaces the MBR/hash value of $N_{4}$ with $\left[P_{5}, P_{6}\right]$. Conceptually, $S C_{\text {new }}$ becomes the $V O$ for query $Q=$ $\left(Q_{1}\right.$ or $\left.Q_{2}\right)$. Case 4 simply appends token entries.

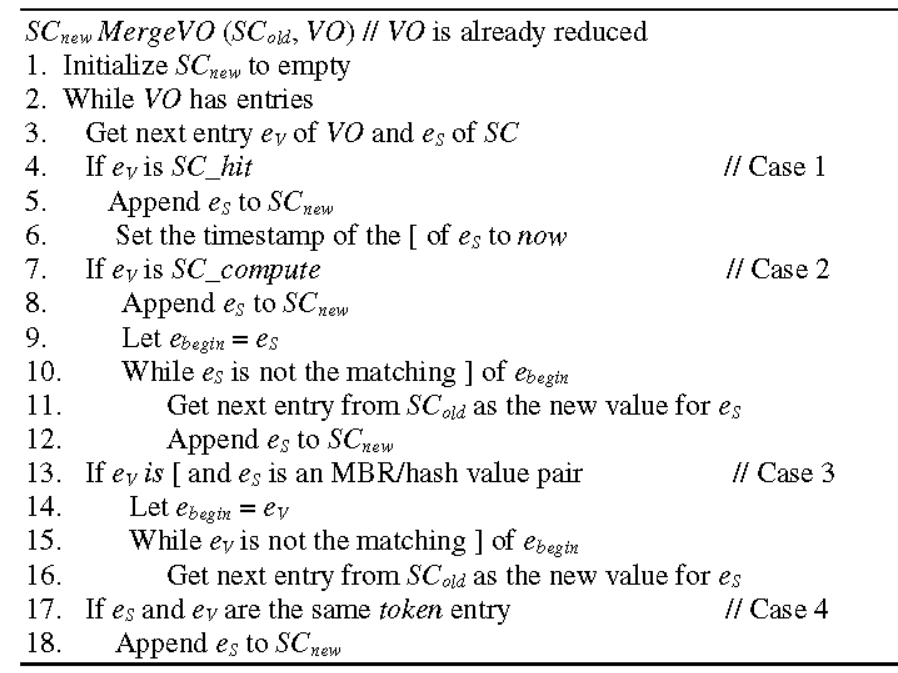

Figure 13 MergeVO algorithm

In our implementation, we assign a limit $L$ to the size of the $S C$ at the client side. $L$ may depend on the memory of the client, or it may be decided by the LBS. In either case, the LBS and the client agree on the value of $L$, which may be different for each client (e.g., the LBS may charge clients according to their cache size). If the $S C$ exceeds $L$ (after an application of MergeVO), PurgeSC (Figure 14) removes the oldest entries to free space. Specifically, PurgeSC performs the opposite of operation MergeVO, i.e., it replaces the details of an MR-node (a sequence of entries bounded by [ and ]) with a single entry that contains the MBR and hash value of the node. The process is applied repeatedly until the size of the $S C$ drops below $L$. At each step, the node to be replaced is chosen according to an LRU policy based on the timestamp 
stored with each [. Recall that these timestamps are maintained by MergeVO.

PurgeSC (SC)

1. While the size of the $S C$ exceeds the limit $L$

2. Scan $S C$ to find the oldest [ that is not enclosed by other tokens. Let $e_{\text {begin }}$ be this [

3. Let $e_{\text {end }}$ be the corresponding ] of $e_{\text {begin }}$

4. Compute the $M B R$ and hash of all $S C$ entries from $e_{\text {begin }}$ to $e_{\text {end }}$

5. Replace all $S C$ entries from $e_{\text {begin }}$ to $e_{\text {end }}$ with a single entry (MBR, hash)

Figure 14 PurgeSC algorithm

\section{EXPERIMENTAL EVALUATION}

We implemented the MR-tree and the VR-tree in $\mathrm{C}++$, using the Crypto++ library [4] and executed all experiments on a P4 $3 \mathrm{GHz}$ CPU. Both MR-tree and VR-tree implementations are based on $\mathrm{R}^{*}$-trees using 4 Kbytes page size. Each experiment is repeated on two datasets: (i) UNI that contains 2 million uniformly distributed data points, and (ii) CAR that contains 2 million points taken from road segments in California [20]. In cases where we want to set a specific cardinality, we randomly sample from these datasets using an appropriate sampling rate. Section V-A compares the initial construction cost and size of MR-trees and VR-trees. Section $\mathrm{V}-\mathrm{B}$ evaluates the query processing and verification overhead of the two structures. Section V-C assesses the benefits of synchronized caching.

\section{A. Initial Construction}

Figure 15 illustrates the construction cost for VR- and MRtrees as a function of the data cardinality. This cost includes both the time to create the trees and the time to compute the hash values (MR-tree) or the signatures (VR-tree). The VRtree is 1-2 orders of magnitude more expensive to build due to the numerous signatures. Figure 16 shows the $\mathrm{CPU}$ time for computing the necessary authentication information. The MRtree outperforms the VR-tree by 3-4 orders of magnitude on this metric. Comparing Figures 16 and 15, the computation of signatures dominates the total construction cost of the VR-tree On the other hand, the MR-tree involves cheap hashing operations, only for the nodes (and not the data points). Consequently, the overhead of the additional information (with respect to the $\mathrm{R}^{*}$-tree) constitutes a small fraction (less than $1 \%$ ) of the total construction cost. Figure 17 illustrates the size of the indexes in MBytes. The VR-tree is much larger since it stores one signature (128 bytes) for each data point and node, where the MR-tree stores one digest (20 bytes) for every node.

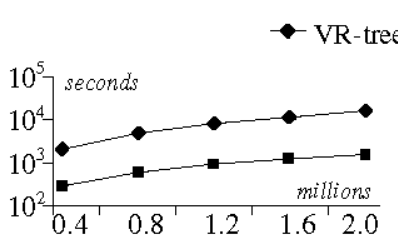

(a) UNI

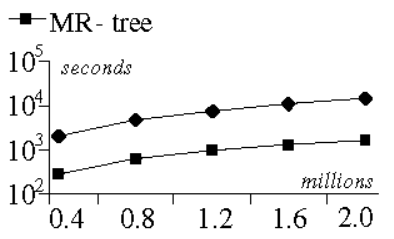

(b) CAR
Figure 15 Total construction time vs. data cardinality

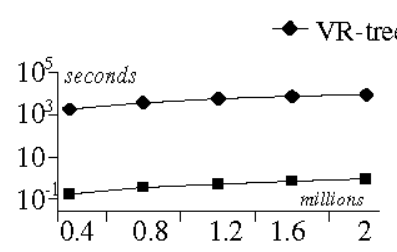

(a) UNI

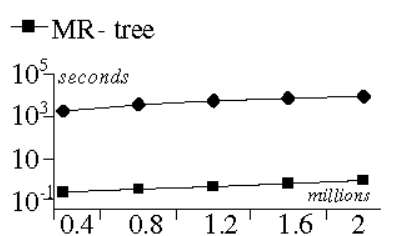

(b) CAR
Figure 16 CPU time for authentication data vs. cardinality

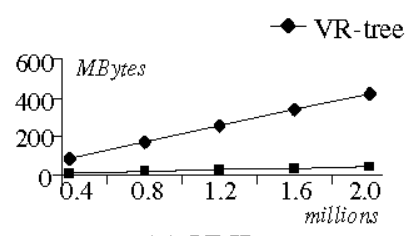

(a) UNI

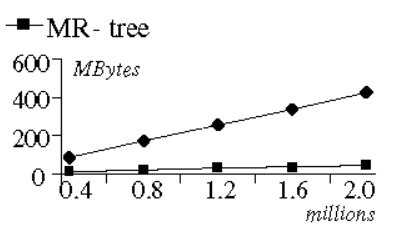

(b) CAR
Figure 17 Index size vs. data cardinality

\section{B. Query Processing and Verification}

This section evaluates the query and verification cost of the two structures. All queries are ranges (recall from Section III, that other query types, such as NN, can be converted to ranges), covering $1 \%$ of the entire (2D) space. For every experiment, we execute 100 ranges at random locations and illustrate the average cost. This cost burdens the LBS and includes both the result retrieval and the construction of the verification object. The data cardinality $(N)$ varies between $0.4 \cdot 10^{6}$ and $2 \cdot 10^{6}$. We do not include synchronized caching since it is evaluated separately in Section V-C.

Figure 18 illustrates the query cost (in seconds) as a function of the data cardinality $\left(Q_{l}=10 \%\right)$. The MR-tree is fast because it creates the $V O$ by simply appending MBRs and hash values for each pruned node. The VR-tree is about 2 orders of magnitude slower due to the modular multiplications required to create the aggregated signature. Recall that signature aggregation is unavoidable because, otherwise, the $V O$ would be extremely large.

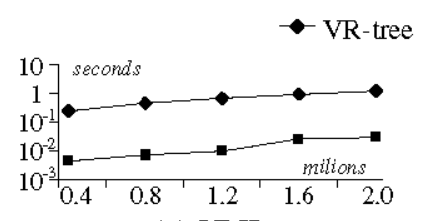

(a) UNI

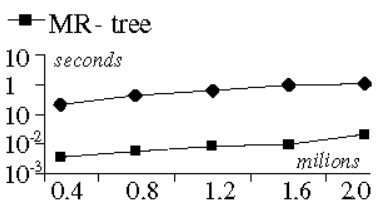

(b) CAR
Figure 18 Query cost vs. data cardinality

Next we measure the verification object. Figure 19 depicts the $V O$ size versus the data cardinality. For small datasets, the $V O$ of MR-trees and VR-trees have similar sizes. However, as the cardinality rises, the $V O$ grows faster for the VR-tree because more intermediate MBRs are included in the $V O$ (due to the smaller fanout). For comparison, the diagrams also illustrate the result size. The verification object (of VR-trees and MR-trees) is larger than the corresponding result, because the result is always part of the $V O$. 


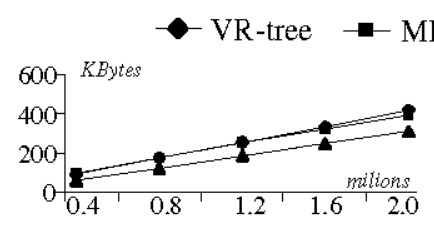

(a) UNI

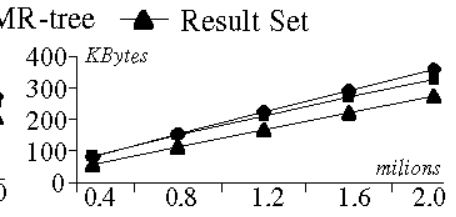

(b) CAR
Figure 19 VO size vs. data cardinality

Finally, Figure 20 investigates the verification time (at the client) versus the data cardinality. The VR-tree leads to high cost since verification involves a number of modular multiplications, which is proportional to the output size. On the other hand, verification in the MR-tree invokes relatively cheap hash operations. Minimization of verification time is crucial for clients (e.g., PDAs) with limited computational resources.

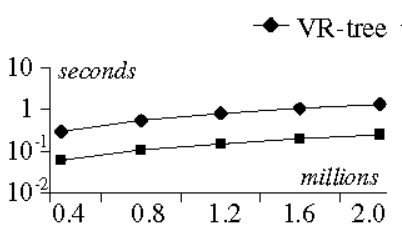

(a) UNI

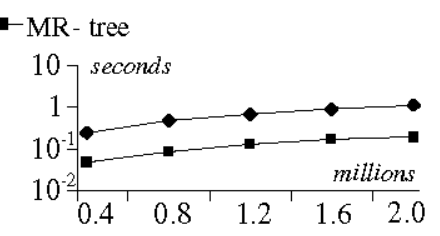

(b) CAR
Figure 20 Verification time vs. data cardinality

Summarizing, the MR-tree is considerably faster to build and consumes less space than the VR-tree. At the same time it is much more efficient for query processing and verification. The only aspect where the MR- and VR-tree have similar performance is the size of the $V O$. Next, we evaluate the impact of synchronized caching on the verification object.

\section{Synchronized Caching}

Recall that synchronized caching entails two caches at the LBS and the client. The $V O$ of each processed query is incorporated in the caches and utilized to reduce the $V O$ of subsequent queries. This optimization is expected to have considerable benefits in cases where successive queries exhibit locality. In our experiments we simulate a moving client that enquires about its surroundings. Specifically, the first query is a range centered at a random location. The user chooses a direction, moves a certain distance and issues another range search (with a fixed extent). The process is repeated 100 times. The $V O$ s of the first 50 queries are used to warm up the cache. For the remaining ones we measure the average reduction achieved per $V O$. The average reduction is defined as $\left(\left|V O_{\text {raw }}\right|-\left|V O_{\text {reduced }}\right|\right) / V O_{\text {raw }} \mid$, where $\left|V O_{\text {reduced }}\right|$ $\left(\left|V O_{\text {raw }}\right|\right)$ is the size of the $V O$ with (without) synchronized caching.

We investigate the effect of the cache size, and the distance traveled between two consecutive queries using the CAR dataset. The results for UNI are similar and omitted due to the lack of space. The data cardinality is set to $2 \cdot 10^{6}$, and the query extent to $10 \%$ per axis. Figure $21 \mathrm{a}$ illustrates the average $V O$ reduction as a function of the cache size (at the client), after fixing the distance between two consecutive queries to $3 \%$ of the axis length. Even $100 \mathrm{KBytes}$ of cache result in a reduction of about $10 \%$. The reduction increases with the cache size and stabilizes at around 500 KBytes. After this point, more cache does not have a significant impact on performance, because the new parts of the tree have to be included in the $V O$ anyway.

The LBS stores, for each client, placeholders for the corresponding hash values and records. Therefore, the cache copy at the LBS consumes much less space than that of the client. The memory consumption per client at the LBS is shown at the bottom of the $x$-axis. Assuming a cache of $500 \mathrm{Kbytes}$ per client (i.e., $1.7 \mathrm{Kbytes}$ at the LBS), a LBS with 1 Gbyte of main memory can support up to $558 \cdot 10^{3}$ clients. If the cache size is 300 Kbytes per client, the LBS can support $833 \cdot 10^{3}$ clients.

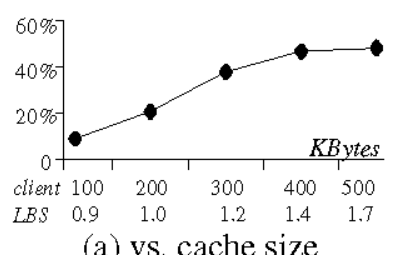

(a) vs. cache size

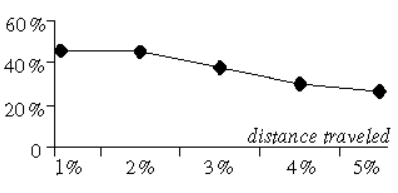

(b) vs. distance traveled
Figure 21 Average $V O$ reduction (CAR)

Figure $21 \mathrm{~b}$ illustrates the average reduction as a function of the distance between successive queries, after setting the cache size to 300 KBytes. As expected, the effect of the cache diminishes with the increasing distance, since the stored $V O$ becomes irrelevant faster. Nevertheless, the distances that we use in these experiments are rather large compared to the locality exhibited in most practical applications.

In conclusion, synchronized caching achieves significant reduction of the $V O$ size, even for small caches and relatively infrequent (or distant) queries. Most potential clients of spatial outsourcing systems (e.g., PDAs) already include flash memory that reaches several Mbytes and could devote part of this memory for caching purposes. The minimization of the verification object, on the other hand, leads to savings in the communication cost, which is very important for wireless networks. Finally, recall that the update algorithms of Section IV eliminate the need to transfer cache information between the LBS and the client.

\section{CONCLUSION}

Recent advances in location based services and sensor networks, as well as the popularity of web-based access to spatial data (e.g., MapQuest, GoogleEarth, etc.), necessitate query authentication for outsourced and replicated multidimensional data. In this paper, we propose the MR-tree, an authenticated index based on the Merkle Hash tree and the $\mathrm{R}^{*}$-tree. Our method outperforms the best current solution by orders of magnitude in many important metrics such as construction cost, index size and verification overhead. Furthermore, we develop a novel synchronized caching protocol, which significantly reduces the communication overhead of the verification step. We conclude our 
contributions with an extensive experimental study that validates the effectiveness and efficiency of the proposed structure.

\section{ACKNOWLEDGMENT}

Yin Yang, Stavros Papadopoulos and Dimitris Papadias were supported by the grant HKUST 6184/06E from Hong Kong RGC. George Kollios was partially supported by the NSF grant IIS-0133825.

\section{REFERENCES}

[1] de Berg, M., van Kreveld, M., Overmars, M., Schwarzkopf, O. Computational Geometry: Algorithms and Applications. Springer-Verlag, 1997.

[2] Beckmann, N., Kriegel, H.-P., Schneider, R., Seeger, B. The $\mathrm{R}^{*}$-tree: An Efficient and Robust Access Method for Points and Rectangles. SIGMOD, 1990.

[3] Cheng, W., Pang, H., Tan, K.-L. Authenticating MultiDimensional Query Results in Data Publishing. DBSec, 2006.

[4] www.eskimo.com/ weidai/benchmark.html

[5] Devanbu, P., Gertz, M., Martel, C., Stubblebine, S. Authentic Data Publication Over the Internet. Journal of Computer Security 11(3): 291-314, 2003.

[6] Guttman, A. R-trees: A Dynamic Index Structure for Spatial Searching. SIGMOD, 1984.

[7] Goodrich M., Tamassia R., Triandopoulos N., Cohen R. Authenticated Data Structures for Graph and Geometric Searching. CT-RSA, 2003.

[8] Hacıguimuiş, H., Iyer, B., Mehrotra, S. Providing Databases as a Service. ICDE, 2002.
[9] Hjaltason, G., Samet, H. Distance Browsing in Spatial Databases. ACM TODS, 24(2):265-318, 1999.

[10] Li, F., Hadjieleftheriou, M., Kollios, G., Reyzin, L. Dynamic Authenticated Index Structures for Outsourced Databases. SIGMOD, 2006

[11] Merkle, R. A Certified Digital Signature. CRYPTO, 1989.

[12] Martel, C., Nuckolls, G., Devanbu, P., Gertz, M., Kwong, A., Stubblebine, S. A General Model for Authenticated Data Structures. Algorithmica, 39(1): 21-41, 2004.

[13] Mykletun, E., Narasimha, M., Tsudik, G. Signature Bouquets: Immutability for Aggregated/Condensed Signatures. ESORICS, 2004.

[14] Menezes, A., van Oorschot, P., Vanstone, S. Handbook of Applied Cryptography. CRC Press, 1996.

[15] Narasimha M., Tsudik G. Authentication of Outsourced Databases Using Signature Aggregation and Chaining. DASFAA, 2006.

[16] Pang, H., Jain, A., Ramamritham, K., Tan, K.-L. Verifying Completeness of Relational Query Results in Data Publishing. SIGMOD, 2005.

[17] Pagel, B., Six, H., Toben, H., Widmayer, P. Towards an Analysis of Range Query Performance in Spatial Data Structures. PODS, 1993.

[18] Papadias, D., Tao, Y., Fu, G., Seeger, B. Progressive Skyline Computation in Database Systems. TODS 30(1), 41-82, 2005.

[19] Pang, H., Tan, K.-L. Authenticating Query Results in Edge Computing. ICDE, 2004.

[20] www.rtreeportal.org

[21] Theodoridis, Y., Sellis, T. A Model for the Prediction of R-tree Performance. PODS, 1996. 